\title{
Having it all
}

\author{
Crystallography has evolved into a vast, multidisciplinary field - but it has enduring significance for \\ physics, and should remain a fixture in university curricula.
}

This year is International Year of

Crystallography (www.iucr2014.org). It marks the centenary of the award of the Nobel Prize in Physics to Max von Laue "for his discovery of the diffraction of X-rays by crystals". Now is clearly a good time to muse on the appeal of crystallography and its relation to physics.

As a discipline, crystallography emerged from the desire to understand and describe the structure of minerals. Today, the study of the arrangements of atoms in solids is still crystallography's core business, but the many analytic tools and experimental methods that it has spawned over the years now pervade practically every branch of the natural sciences - crystallographers come disguised as mathematicians, physicists, materials scientists, chemists and biologists. But crystallography can reach still further, proving useful, for example, to art historians in understanding the intricate patterns of medieval Islamic art ${ }^{1}$.

Although the field of crystallography is now sufficiently mature to perhaps be perceived as dull, or 'finished', the reality is that it is in continuous development. In 1992, the International Union of Crystallography (IUCr) redefined ${ }^{2}$ the very word 'crystal' to accommodate aperiodic crystals (such as the quasicrystals that were discovered in 1984): "a material is a crystal if it has essentially a sharp diffraction pattern". The International Tables for Crystallography the ultimate series of crystallography handbooks published under the auspices of the IUCr (http://it.iucr.org) - have been constantly updated and expanded since their first edition in 1952. And earlier this year, Nature Physics published remarkable new findings ${ }^{3}$ on the relation between spin-orbit coupling effects and crystal symmetry.

Today, not only has our ability to characterize crystalline materials advanced, but so has our capability to study noncrystallinity - be it in the form of local defects in otherwise perfect crystals, or amorphous and soft matter such as glasses, gels, liquid crystals or polymers. In reaching for perfection, one learns much about imperfection.

Nature Publishing Group encompassing Nature, Nature Physics and its sister titles - has contributed a special supplement to the celebrations of the International Year of Crystallography: Nature
Milestones in Crystallography tells the history of the field in 25 instalments, each recounting a major breakthrough and together illustrating the broad scope and influence of the discipline. The supplement is available online, with an extensive library of material from across the Nature Publishing Group, at www.nature.com/milestones - and is accessible for free for a six-month period.

The link between crystallography and solid-state physics, or more modernly, condensed-matter physics, is obvious. In a way, crystallography is to solid-state physics what mathematics is to the whole of physics - a language, with vocabulary and grammar, there to describe the physical world. And yet, although extensive, selfcontained training in pure mathematics for students of physics has always, rightly, been considered essential, the situation is completely different for crystallography.

All too often, the curricula followed by university physics students don't feature a crystallography course: rather, the subject is typically crammed into a few introductory chapters of a solid-state physics course. That's a pity. There are a good many reasons for future physicists to study a stand-alone, indepth crystallography module or programme. To start with, there is the sheer breadth of the subject. It takes time to fully appreciate and understand the subtleties of space groups; add in reciprocal space and diffraction (theory and experiment), plus incommensurate crystals, and your semester is already quite packed.

One major benefit of teaching crystallography as a separate subject is that it is a useful vehicle for introducing concepts that are common to so many branches of physics - symmetry, group theory, k-space, Fourier transforms, and scattering, to name but a few. Again, many of these concepts have particular relevance to solid-state physics (electronic band structure theory and the principles of lattice dynamics both heavily rely on reciprocal space, for example), but you'll also encounter them in basic quantum theory or the standard model of particle physics.

A 'quick' introduction to crystallography simply doesn't pay off in the long run. Not all is cubic, mono-atomic, single-phase or defect-free. And although table-top X-ray diffractometers are now available that, at the push of a button, will give you the structure of a well-behaved sample within minutes,

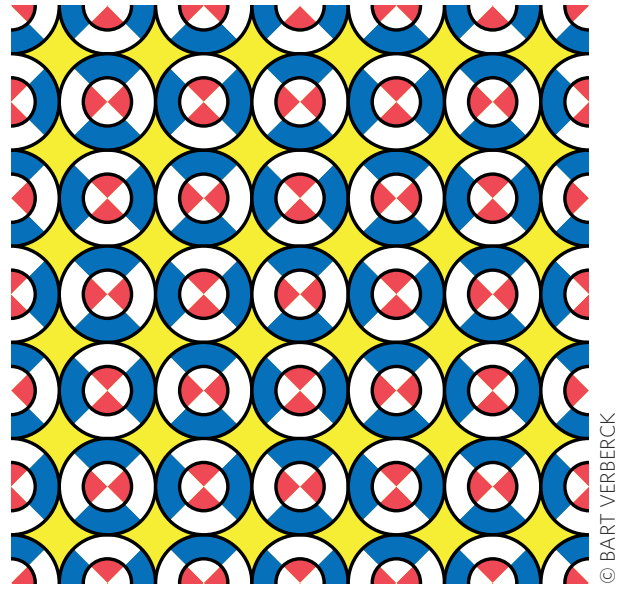

A planar tiling: but what is its 2D space group?

you're then on your own in attempting to understand why the structure is what it is, and what its physical implications (structureproperty relationships) are. Indeed, to make the point that crystallography is perhaps not as straightforward as it may seem, here's a teaser: consider the pattern pictured here, and imagine it replicated infinitely horizontally and vertically. Try figuring out its 2D space group (or 'wallpaper' group) it's rather subtle.

The importance of crystallography, and its teaching, is - naturally - recognized by the IUCr. This International Year has many events jointly organized by the IUCr and UNESCO to promote crystallography, both for the general public and the scientific community. The IUCr also seeks to foster international collaboration: IUCr-UNESCO OpenLab is a new project aimed at boosting access to crystallographic knowledge and facilities for scientists all over the world.

Crystallography is as exciting as ever, and worth investing in - as the participants at the 23rd Congress and General Assembly of the IUCr, taking place this month in Montreal, Canada, will surely agree. (And if you happen to be there, pick up a free print copy of Nature Milestones in Crystallography!)

References

1. Lu, P. J. \& Steinhardt, P. J. Science 315, 1106-1110 (2007).

2. International Union of Crystallography, Report of the Executive Committee for 1991 Acta Cryst. A 48, 922-946 (1992).

3. Zhang, X., Liu, Q., Luo, J.-W., Freeman, A. J. \& Zunger, A. Nature Phys. 10, 387-393 (2014). 